\title{
Modeling of Tunneling Current in Bilayer Armchair Graphene Nanoribbon-Tunneling Field Effect Transistor by Using Airy Wave Function Approach
}

\author{
Shofi Dhiya 'Ulhaq', Muhammad Fulki Fadhillah², Amelia Fadhillah', Intan Anjaningsih", \\ Andhy Setiawan ${ }^{5}$, Endi Suhendi ${ }^{6}$ \\ \{endis@upi.edu\}
}

Program Studi Fisika, Universitas Pendidikan Indonesia, Bandung 40154, Indonesia ${ }^{1,2,3}$

\begin{abstract}
Metal oxide semiconductor field-effect transistor (MOSFET) has an important role in electric circuits because of its nano-size. However, MOSFETs have performance limitations to work in ultra-low power, leaky currents, short channel effects, and increased speed. These performance limitations make the tunneling field-effect transistor (TFET) an alternative to replace the MOSFET. In this study, a drain current on devices based on bilayer armchair graphene nanoribbon (BAGNR) was modeled using the Airy function approach. Solving the Schrödinger equation with the Airy function approach produces a transmittance value, and therefore the drain current value can be determined using the Launder formula with the Gauss Legendre Quadrature (GLQ) method. The results of the modeling show that the drain current value increases with the increasing gate voltage $(\mathrm{Vg})$, drain voltage $(\mathrm{Vd})$ and width of BAGNR, while the increase in oxide thickness and temperature decreases the drain current.
\end{abstract}

Keywords: Airy Wave Function, Tunneling Current, BAGNR, TFET

\section{Introduction}

T. Baba developed the tunneling field-effect transistor (TFET) since 1992 [1]. TFET has more advantages than MOSFETs in the needs of electronic applications. The advantages of using TFET are: suitable for low power applications due to the lower possibility of leakage, the ability to work at sub-threshold and super-threshold voltages, similarity in the MOSFET fabrication process and increased operating speed due to the principle of tunneling [2,3]. By using the principle of tunneling, TFET will be more effective if it uses a material that has a high mobility of the charge carrier. Mobility of charge carriers in graphene material can reach 15,000 $\mathrm{cm}^{2} / \mathrm{Vs}$ and can be increased up to $\approx 100,000 \mathrm{~cm}^{2} / \mathrm{Vs}[4]$.

Graphene was discovered in 2004 by Konstantin Novoselov and Andre Geim by using what would be later known as the Scotch - tape method. Monolayer graphene and bilayer graphene have energy band gap values of around zero. To compare the energy of the band structure on the monolayer and bilayer graphene can be applied with the Tight Binding Model [5]. The results show the structure of the electronic energy band in bilayer graphene is low than monolayer graphene. Another advantage of bilayer graphene is that it can induce a band gap and regulate its magnitude by using a strong electric field perpendicular to the carbon layer [6].

The research conducted is a tunneling current of the BAGNR-based TFET device by solving the Schrödinger equation with the Airy function method approach. With the same 
method, TFET research with monolayer graphene was carried out [7]. This approach is a semianalytical method to solve the problem of tunneling currents which has been shown to have a high accuracy compared to the Wentzel-Kramers-Brillouin (WKB) approach [8]. The weakness of the WKB method is the discontinuity at the turning point [9].

\section{Methods}

The calculation of BAGNR energy band gap quantized by using equation (1) [6]:

$$
E_{g}=\frac{\hbar^{2} \pi^{2} v_{f}^{2}}{9 w^{2} \gamma_{1}}
$$

where $\mathrm{w}$ is the width of the bilayer graphene armchair, $\gamma_{1}$ is an interlayer coupling that has a value $\approx 0,39 \mathrm{eV}[10]$ and $v_{f}$ is the speed of the fermi.

The TFET potential profile function is obtained by solving the Poisson equation by entering its boundary conditions [11,12]. Potential energy for each region in BAGNR TFET are shown in Figure 1.

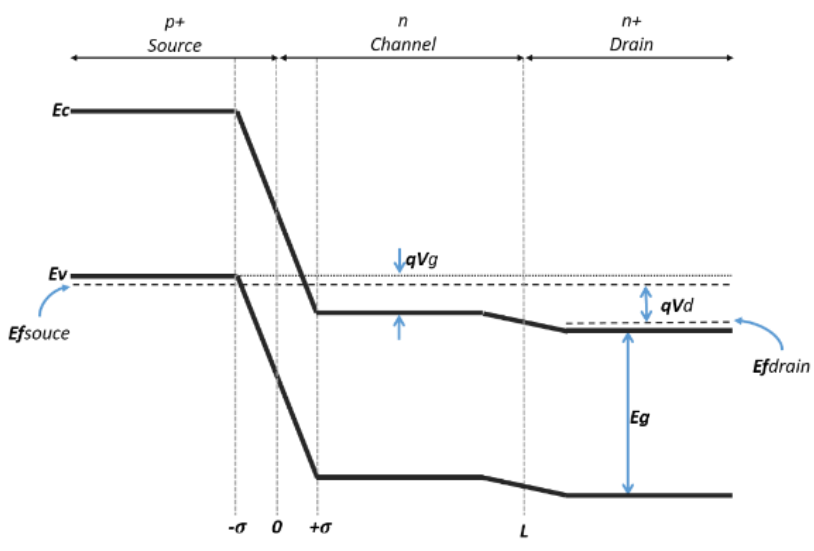

Fig. 1. Potential energy of BAGNR TFET model under the voltage application to the gate.

Here, Ef is the Fermi energies. Ec and Ev are the conduction and valence of the band. Eg is the energy gap. $\mathrm{Vg}$ and $\mathrm{Vd}$ are the gate and drain voltages, $\mathrm{q}$ is the electronic charge. $\mathrm{L}$ is the length of the channel.

The free-electron wave function in each region in BAGNR TFET potential energy is a solution of the Schrödinger equation. Analytically it can be described in the form of Airy's function solution. To get the solution of the wave function to position $(\psi(x))$, the Airy function method is used. The Airy function is as shown in equation (2):

$$
\frac{d^{2} \psi}{d \zeta^{2}}-\zeta \psi(\zeta)=0
$$


The PDB solution is written in the two forms of Airy functions that are linearly independent of each other, $\operatorname{Ai}(\zeta)$ and $B i(\zeta)$ as shown in equation (3):

$$
\psi=C A i(\zeta)+D B i(\zeta)
$$

where $\mathrm{C}$ and D are Airy's function constants.

Tunneling current is the probability of electrons to move through potential barriers. Formulae to find out tunneling currents on the GNR TFET assuming ballistic transportation occurs in the channel, the transmittance obtained is then applied to calculate tunneling currents using the Launder equation [7] as shown in equation (4):

$$
I=\frac{2 q}{\pi \hbar} \int_{E_{c c}}^{E_{v s}}\left[f_{S}(E)-f_{D}(E)\right] T(E) d E
$$

Under the condition of the integral limit of the energy of the valence band electron $\left(E_{v s}\right)$ to the conduction band electron energy $\left(E_{c c}\right), f_{S}(E)$ and $f_{D}(E)$ is the Fermi-Dirac distribution function for source and drain electrons, written as follows: $f_{S}(E)=\frac{1}{1+\exp \left(\frac{E+e V_{D}}{k_{B} T}\right)}$ and $f_{D}(E)=$ $\frac{1}{1+\exp \left(\frac{E}{k_{B} T}\right)} . k_{B}$ is Boltzmann constant, $\hbar$ is Planck constant, and $T(E)$ is a transmission coefficient.

\section{Result and Discussion}

Figure 2 shows the tunneling current to the drain voltage with gate voltage variations. The parameters used for channel length, $\mathrm{N}$ index of BAGNR, oxide thickness, and temperature are $20 \mathrm{~nm}, 25,1 \mathrm{~nm}$ and $300 \mathrm{~K}$ respectively. Meanwhile, Figure 3 shows the tunneling current to the drain voltage with variations in the $\mathrm{N}$ index of BAGNR. The parameters used for channel length, oxide thickness, gate voltage, and temperature are respectively $20 \mathrm{~nm}, 1 \mathrm{~nm}, 0.15 \mathrm{~V}$ and $300 \mathrm{~K}$.

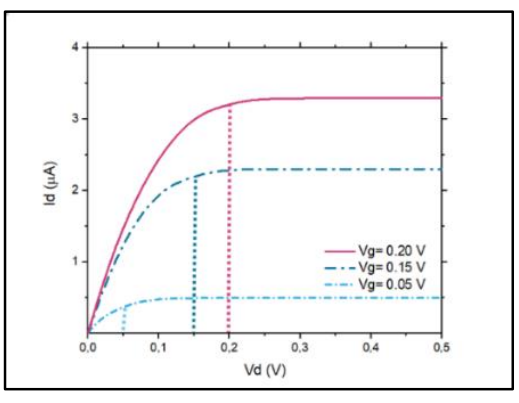

Fig. 2. Tunneling current graph to the drain voltage with variations of gate voltage.

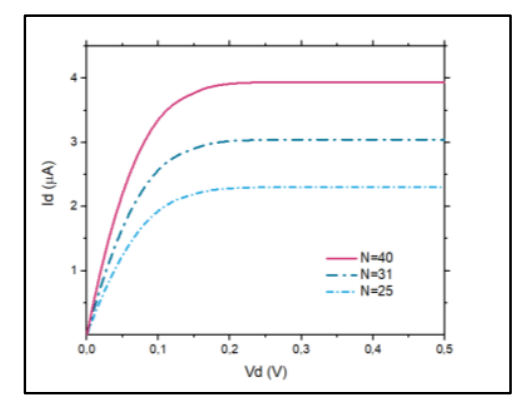

Fig. 3. Tunneling current graph to the drain voltage with variations of $\mathrm{N}$ index.

Figure 2 shows that the tunneling current increases with the increasing value of the drain voltage $(\mathrm{Vd})$ until it reaches its maximum value like the other researches that have been done 
before $[7,13,14]$. After the tunneling current reaches a maximum, the current is then constant or called saturation. Physically, the condition occurs when the edges of the drain conduction band are the same or lower than the conduction band channel. A high gate voltage value $(\mathrm{Vg})$ causes the channel energy band to decrease while a high value of drain voltage $(\mathrm{Vd})$ makes the drain energy band decrease. When the channel conduction band gets lower, electrons easily flow from source to channel. This gives an increase in the number of electrons in the channel. When the drain conduction band has the same value as the channel conduction band, electrons flow without being blocked by potential drain energy bands, making it easy to flow from source to drain. But when the drain conduction band has a lower value than the conduction band channel, the number of electrons flowing does not increase. The amount will be the same as the number of electrons in the channel. So, that causes the current no longer increases, and produces a constant value called saturation.

The $\mathrm{N}$ index value of the BAGNR is proportional to the width of the BAGNR (w), the greater the value of $\mathrm{N}$ then the width of the BAGNR is increasing. This affected the energy band gap (Eg) which is inversely proportional to the width of the BAGNR. The small energy band gap (Eg) makes it easier for electrons to break through the barrier. So that is why Figure 3 shows the tunneling current increases when the $\mathrm{N}$ index increases, like the other researches that have been done before $[15,16]$.

Figure 4 and Figure 5 show the tunneling current to the drain voltage. Figure 4 uses the variations of oxide thickness. The parameters used for channel length, $\mathrm{N}$ index and temperature are $20 \mathrm{~nm}, 25$ and $300 \mathrm{~K}$. Meanwhile, Figure 5 uses the variations of temperature variations. The parameters used for channel length, oxide thickness and $\mathrm{N}$ index are $20 \mathrm{~nm}, 1 \mathrm{~nm}$ and 25.

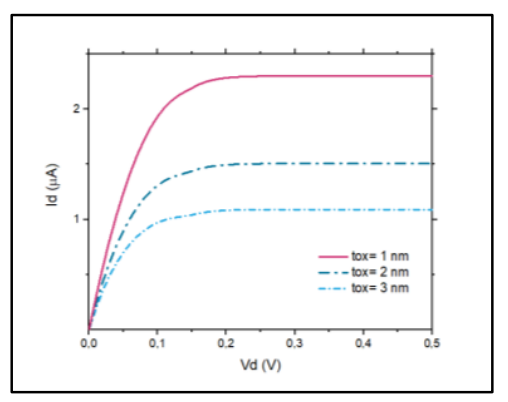

Fig. 4. Tunneling current graph to the drain voltage with variations of oxide thickness.

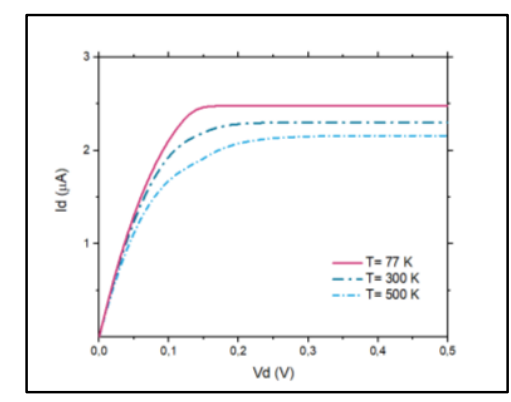

Fig. 5. Tunneling current graph to the drain voltage with variations of temperature.

Figure 4 shows that the tunneling current decrease when the oxide thickness increase like the other research that has been done before [17]. Decreased tunneling current with the increasing oxide thickness due to thick oxide affecting gate capacitance [18]. Total gate capacitance can be expressed as a series of insulator (oxide) capacitance and inversion layer capacitance [19]. Whereas oxide capacitance is inversely proportional to its thickness [20]. So that the thicker the oxide used, the capacitance decreases so that gate capacitance decreases as well. When the gate capacitance decreases the electrons flowing in the channel decreases and so the value of the tunneling current. In addition, the thickness of the oxide affects the width of the depletion; the thicker the oxide used, the wider the depletion. For $1 \mathrm{~nm}$ oxide thickness, the width of depletion that the device will have is $1.18 \mathrm{~nm}$. For $2 \mathrm{~nm}$ and $3 \mathrm{~nm}$, thick oxides respectively are $1.67 \mathrm{~nm}$ and $2.05 \mathrm{~nm}$. Large depletion width reduces the ability of electrons to break through the barrier wall. 
Figure 5 shows when TFET is at a low temperature, the tunneling current is higher because the charge mobility highly increased like the other researches that have been done before $[17,21]$. Whereas at high temperatures, the charge mobility is low so that tunneling currents are lower compared to low temperature conditions. Tunneling current decreases with the increasing temperature because an increase in temperature causes a decrease in charge mobility in the channel and affects its speed [22]. High temperatures make the charge have a large kinetic energy and make it move randomly. When the random charge movement takes place, actual charge mobility is low. Low charge mobility reduces charge speed, resulting in lower tunneling currents than at low temperatures. The energy between the Fermi energy $E_{f}$ and the source valence energy $\mathrm{E}_{\mathrm{vs}}$ band is the charge multiplied by the thermal potential $\mathrm{qV}_{\mathrm{T}}$ or equivalent to $k_{B} T$ [23]. When the higher temperature is applied, the thermal potential is greater and makes the energy between $E_{\mathrm{f}}$ and $\mathrm{E}_{\mathrm{vs}}$ greater. The higher the temperature of the situation, the higher the electron energy required to pass through the barrier potential. If the electron energy is the same, the transmittance value will be higher at a low-temperature state than at a hightemperature state. In addition, temperature affects the Fermi-Dirac distribution on the source and drain. In a high-temperature state, some electrons located slightly below the Fermi energy get enough energy to move to a higher energy level than the Fermi energy. So the chance of finding electrons at a lower energy level than the Fermi energy will be smaller than in a low temperature state.

Tunneling current with gate voltage with variations in drain voltage is shown in the graph in Figure 6(a). The tunneling current increased when the gate voltage increases like the other research that has been done before [18]. In the graph, all three curves generated from variations in drain voltage $(\mathrm{Vd}) 0.01 \mathrm{~V}, 0.05 \mathrm{~V}$ and $0.10 \mathrm{~V}$ have linear characteristics in the voltage range gate $(\mathrm{Vg})$ 0-0,1 V shown by Figure 6(b).

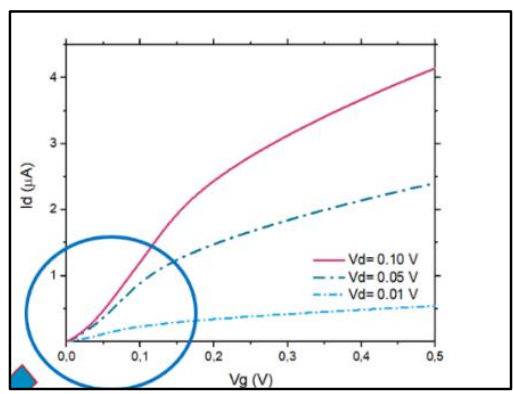

(a)

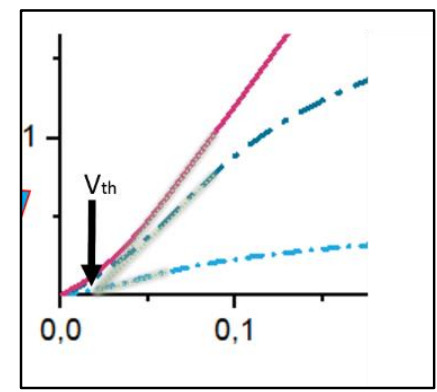

(b)

Fig. 6. (a) Tunneling current graph to the gate voltage (b) The linear characteristics in tunneling current graph to the gate voltage.

From the graph in Figure 6(b), this threshold voltage can be obtained by extrapolating the linear line from the tunneling current graph to the gate voltage $(\mathrm{Vg})$ until it intersects to the $\mathrm{x}$ axis. After being extrapolated, the linear lines go to the same point. This shows the threshold voltage (Vth) with a variation of the drain voltage $(\mathrm{Vd})$ has the same value, which is $0.02 \mathrm{~V}$. It is larger than in AGNR TFET [24]. 


\section{Conclusion}

The tunneling current to the drain voltage produces a graph that can show the saturation region which is affected by the gate voltage. $\mathrm{N}$ index, oxide thickness, and temperature affect the tunneling current. The drain current value increases with the increasing gate voltage (Vg), drain voltage $(\mathrm{Vd})$ and width of BAGNR, while the increase in oxide thickness and temperature decreases the drain current. The tunneling current to the drain voltage produces a graph that can show the threshold voltage as well.

\section{References}

[1] Toshio, B.: Proposal for Surface Tunnel Transistors. Jpn. J. Appl. Phys., vol. 31, no. Part 2, No. 4B, pp. L455-L457 (1992)

[2] Aswathy, M., Biju, N.M., and Komaragiri, R.: Comparison of a 30nm tunnel field effect transistor and CMOS inverter characteristics. Proc. - 2013 3rd Int. Conf. Adv. Comput. Commun. ICACC 2013, pp. 149-152 (2013)

[3] Turkane, S.M. and Kureshi, A.K.: Review of tunnel field effect transistor (TFET). Int. J. Appl. Eng. Res., vol. 11, no. 7, pp. 4922-4929 (2016)

[4] Geim A.K. and Novoselov, K.S.: The rise of graphene. Nat. Mater. (2007)

[5] Menacho, L., Carrasco, M. and Ayala, Z.: Comparison between Monolayer and Bilayer Graphene energy bands using the Tight Binding model. J. Phys. Conf. Ser., vol. 1143, no. 1 (2018)

[6] Russo, S., Craciun, M.F., Khodkov, T., Koshino, M., Yamamoto, M. and Tarucha, S.: Electronic transport properties of few-layer graphene materials. INTECH Open Access Publ. (2011)

[7] Bimo, C.S.P., Noor, F.A., Abdullah, M. and Khairurrijal: A Theoretical Model of Band-to-Band Tunneling Current in an Armchair Graphene Nanoribbon Tunnel Field-Effect Transistor. Adv. Mater. Res. (2014)

[8] Oktasendra, F.: Pengaplikasian Metode Fungsi Airy pada Permasalahan Probabilitas Terobosan Kuantum. J. Ilmu Fis. Indones., vol. 2, no. 2, pp. 75-80 (2016)

[9] Smith A. and Baghai-wadji, A.: A Numerical Technique for Solving Schrödinger's Equation in Molecular Electronic Applications. Proc. SPIE - Int. Soc. Opt. Eng. (2008)

[10] Neto, A.H.C., Guinea, F., Peres, N.M.R., Novoselov, K.S., and Geim, A.K.: The electronic properties of graphene. Rev. Mod. Phys., vol. 81, no. March (2009)

[11] Zhang, Q., Fang, T., Xing, H., Seabaugh, A., and Jena, D.: Graphene nanoribbon tunnel transistors. IEEE Electron Device Lett., vol. 29, no. 12, pp. 1344-1346 (2008)

[12] Sustini, E., Syariati, R. and Khairurrijal : TFET I-V characteristics made of bi layer Armchair Grafene Nano Ribbon (AGNR). J. Phys. Conf. Ser., vol. 1120, p. 012095 (2018)

[13] Syariati, R., Suhendi, E., Noor, F.A., and Khairurrijal, K.: Simulation of Tunnel Current in an Armchair Graphene Nanoribbon-Based p-n Diode for Undergraduate Physics Students. Int. Conf. Adv. Educ. Technol., no. Icaet, pp. 136-139 (2015)

[14] Suhendi, E., Noor, F.A., Kurniasih, N. and Khairurrijal : Modeling of Drain Current in Armchair Graphene Nanoribbon Field Effect Transistor Using Transfer Matrix Method. Adv. Mater. Res., vol. 896, pp. 367-370 (2014)

[15] Suhendi, E., Syariati, R., Noor, F.A., Kurniasih, N. and Khairurrijal, K.: Modeling of Dirac Electron Tunneling Current in Bipolar Transistor Based on Armchair Graphene Nanoribbon Using a Transfer Matrix Method. Adv. Comput. Sci. Res., vol. 2, no. September, pp. 165-167 (2014)

[16] Suhendi, E., Syariati, R., Noor, F.A, Kurniasih, N. and Khairurrijal: Simulation of Dirac Tunneling Current of an Armchair Graphene Nanoribbon-Based P-N Junction Using a Transfer Matrix Method. Adv. Mater. Res., vol. 974, pp. 205-209 (2014) 
[17] Suhendi, E., Hasanah, L., Rusdiana, D., Noor, F.A., Kurniasih, N. and Khairurrijal, K.: Comparison of tunneling currents in graphene nanoribbon tunnel field effect transistors calculated using Dirac-like equation and Schrödinger's equation. J. Semicond (2019)

[18] Suhendi, E., Syariati, R., Noor, F.A., Kurniasih, N. and Khairurrijal : Simulation of drain currents of double-gated armchair graphene nanoribbon field-effect transistors by solving Dirac 'like' equation and using transfer matrix method. J. Phys. Conf. Ser., vol. 539, no. 1 (2014)

[19] Takagi S.I. and Toriumi A.: Quantitative Understanding of Inversion-Layer Capacitance in Si MOSFET's. IEEE Trans. Electron Devices, vol. 42, no. 12, pp. 2125-2130 (1995)

[20] Sanjeet, K.S. and Saurabh, C.: Impact of Oxide Thickness on Gate Capacitance - A Comprehensive Analysis on MOSFET, Nanowire. IEEE Trans. Nanotechnol., vol. 12, no. 6, pp. 958964 (2013)

[21] Suhendi, E., Syariati, R., Noor, F.A., Kurniasih, N. and Khairurrijal : Model of a tunneling current in a p-n junction based on armchair graphene nanoribbons - An Airy function approach and a transfer matrix method. AIP Conf. Proc., vol. 1589, pp. 91-94 (2014)

[22] Djelti, H., Feham, M., Ouslimani, A. and Kasbari, A.: Temperature effects on the Drain Current in GaN Dual-Gate MESFET using Two-Dimensional Device Simulation. Int. J. Comput. Sci. Issues, vol. 9, no. 1, pp. 126-129 (2012)

[23] Sustini, E., Khairurrijal, Noor, F.A., and Syariati, R.: Band Gap Calculations of Bilayer Graphene and Bilayer Armchair Graphene Nanoribbon. IOP Conf. Ser. Mater. Sci. Eng., vol. 367, no. 1 (2018)

[24] Suhendi, E., Hasanah, L., Noor, F.A. and Kurniasih, N.: Modeling of Armchair Graphene Nanoribbon Tunnel Field Effect Transistors for Low Power Applications," J. Semicond. Technol. Sci., vol. 19, no. 4, pp. 336-346 (2019) 